\title{
DIFERENCAS SEXUAIS E RESULTADO CLÍNICO FUNCIONAL APÓS ADMISSÃO POR ACIDENTE VASCULAR CEREBRAL
}

\section{Larissa Mariana Oliveira Santos, Ana Railka de Souza Oliveira Kumakura.}

\section{Resumo}

Entender as variáveis que expliquem diferenças epidemiológicas na incidência e gravidade do Acidente Vascular Cerebral (AVC) podem ser úteis na identificação de grupos específicos com um risco aumentado e nas propostas futuras a serem consideradas como recomendações de prevenção primária e secundária. Objetivo: avaliar o processo de cuidado de pacientes com AVC, verificar a associação entre fatores de risco modificáveis e não modificáveis e as características específicas do sexo e os desfechos clínicos funcionais. Método: Coorte prospectiva, com acompanhamento de pacientes que realizaram atendimento da fase aguda desde outubro de 2017 ( $N=47$ ). Coleta de dados do tipo primária e secundária, com utilização de escalas que medem a funcionalidade prévia ao AVC e o impacto do AVC. Realizou-se estatística descritiva e aplicou-se testes inferenciais, de associação e comparação por meio do SPSS, versão 24.0. Resultados: Os fatores de risco ligados ao sexo encontrados com maior frequência foram os femininos: gravidez $\geq 3$, menarca $\leq 12$ anos, menopausa $\leq 55$ anos e uso de ACO. A média da escala de Frenchay: 23,00; Média do NIH de chegada: 8,27; Média do NIH de alta: 2,56. Não houve associação estatísticas entres as variáveis sociodemográficas e clínicas com os fatores de risco específicos por sexo e os desfechos funcionais. Conclusão: Estudos de acompanhamento dos desfechos clínicos e funcionais dos pacientes com AVC são fundamentais, ao se considerar a mudança nos perfis epidemiológicos e o sugirmento de novos fatores de risco.

Palavras-chave: acidente vascular cerebral; fatores de risco; capacidade funcional.

\section{Introdução}

A população acometida pelo AVC apresenta inúmeras complicações de saúde que corroboram para os déficits de autocuidado, nesse contexto, estudar os fatores de risco é recomendado, principalmente após pesquisas apontarem 0 aumento do número absoluto de sobreviventes a cada ano, características por sexo que aumentam o risco de AVC e também encontrarem associação entre estilo de vida, gravidade do AVC e mortalidade diferindo de acordo com o sexo ${ }^{1,2}$.

Objetivos: Investigar os fatores de risco modificáveis e não modificáveis; Investigar as características específicas do sexo que influenciam desfechos adversos nos resultados clínicos funcionais; Avaliar o processo de cuidado; Verificar a associação entre as variáveis sociodemográficas, clínicas e funcionais;

\section{Resultados e Discussão}

Tabela 1. Resultados clínico funcionais

\begin{tabular}{lllll}
\multicolumn{1}{c}{ Escalas } & $\mathbf{n}^{*}$ & Média & DP† & \multicolumn{1}{c}{ Mín - Máx } \\
\hline NIH chegada & 30 & 8,27 & 6,78 & $0,00-22,00$ \\
NIH alta & 18 & 2,56 & 4,29 & $0,00-18,00$ \\
Rankin chegada & 17 & 0,47 & 1,01 & $0,00-4,00$ \\
Rankin alta & 12 & 1,00 & 1,47 & $0,00-4,00$ \\
Frenchay & 39 & 23,00 & 8,24 & $3,00-41,00$
\end{tabular}

${ }^{*}$ Missing (dados não constavam no prontuário ou paciente não aceitou responder); †desvio-padrão.

Tabela 2. Caracterização dos pacientes com AVC quanto aos fatores risco modificáveis e não modificáveis. ( $N=47)$. Campinas, 2018

\begin{tabular}{lcc}
\hline \multicolumn{1}{c}{ Fatores de Risco } & N & $\%$ \\
\hline Hipertensão Arterial Sistêmica & 35 & 74,5 \\
Dislipidemias & 21 & 44,7 \\
Sedentarismo & 20 & 42,6 \\
Uso de AAS & 19 & 40,4 \\
Ex-tabagista & 25 & 53,2 \\
Etilismo Atual & 15 & 31,9 \\
Ex-etilista & 12 & 25,5 \\
\hline
\end{tabular}

Figura 2. Caracterização dos pacientes com AVC quanto aos fatores de risco ligados ao sexo. ( $\mathrm{N}$ feminino=23; $\mathrm{N}$ masculino=24). Campinas, 2018

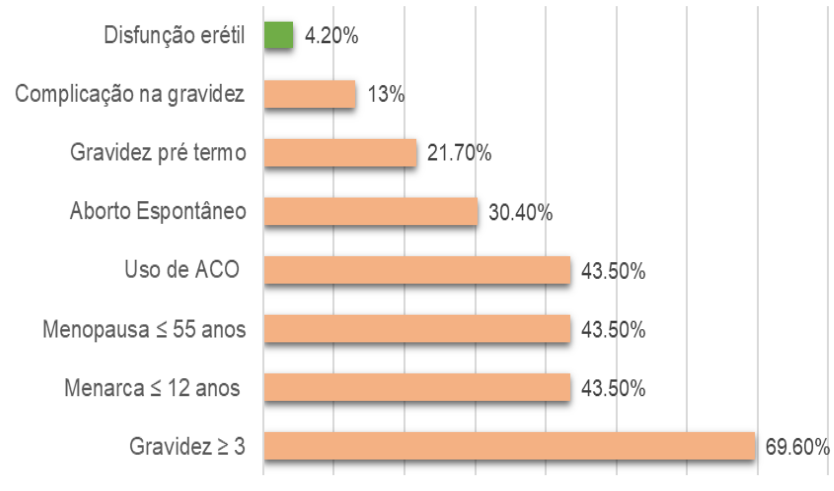

Testes de associação e correlação entre as variáveis não apresentaram significância estatística. Conclusões

Os fatores de risco ligados ao sexo feminino mostraramse mais frequentes dentre as pacientes que foram acometidas pelo AVC. Os prontuários dos pacientes não estavam completos, principalmente no registro das escalas que medem o impacto do AVC na vida funcional do paciente (NIH e Rankin). Isso dificulta o acompanhamento da progressão funcional e até mesmo o início precoce do processo de reabilitação.

\section{Agradecimentos}

Agradecimentos ao $\mathrm{PIBIC} / \mathrm{CNPq}$ pelo auxílio financeiro através da bolsa de Iniciação Científica

1 Vangen-Lønne AM, Wilsgaard T, Johnsen SH, Løchen ML, Njølstad I, Mathiesen EB. Declining Incidence of Ischemic Stroke. What Is the Impact of Changing Risk Factors? The Troms $\varnothing$ Study 1995 to 2012. Stroke. 2017; 48:544-550. DOI: 10.1161/STROKEAHA.116.014377. ${ }^{2}$ Poorthuis MHF, Algra AM, Algra A, Kappelle LJ, Klijn CJM. Female- and Male-Specific Risk Factors for Stroke. A Systematic Review and Meta-analysis JAMA Neurol. 2017;74(1):75-81. doi:10.1001/jamaneurol.2016.3482 